\title{
Anatomía de la Cabeza Femoral Humana: Consideraciones en Ortopedia, Parte II. Biomecánica y Morfología Microscópica
}

\author{
Anatomy of the Human Femoral Head. Orthopedic Issues, Part II. \\ Biomechanics and Microscopic Morphology
}

*Raúl Álvarez San Martín \& ***osé Antonio Velutini Kochen

\begin{abstract}
ÁLVAREZ, S. M. R. \& VELUTINI, K. J.A. Anatomía de la cabeza femoral humana. Consideraciones en ortopedia, Parte II. Biomecánica y morfología microscópica. Int. J. Morphol., 29(2):371-376, 2011.

RESUMEN: Las variantes anatómicas microscópicas y las características biomecánicas de la cabeza femoral son aspectos que han servido como sustento científico para el entendimiento de la vasta patología que afecta a la articulación coxofemoral y el desarrollo tanto de implantes de reemplazo articular como de técnicas de reconstrucción ósea. En esta revisión se exponen los aspectos morfológicos microscópicos y biomecánicos de la cabeza femoral, en relación al extremo proximal del fémur, para describir las características anatómicas microestructurales y funcionales que hacen de ésta, uno de los sitios anatómicos de mayor importancia en la cirugía ortopédica.
\end{abstract}

PALABRAS CLAVE: Cabeza femoral; Fémur proximal; Microestructura; Biomecánica; Ortopedia.

\section{INTRODUCCIÓN}

La microestructura y la biomecánica de la cabeza femoral han permitido realizar varias correlaciones con diversas patologías que afectan a la cadera. En este artículo se plantea actualizar al lector con las características microestructurales y biomecánicas de la cabeza femoral.

Sistema óseo trabecular. La estructura trabecular interna del fémur proximal fue descrita por Ward, en 1838. De acuerdo con la ley de Wolf, las trabeculaciones surgen sobre las líneas de fuerza a las que el hueso está expuesto. En el cuello femoral y en la región intertrocantérica, la trabeculación presenta una transición desde la corteza ósea hacia la metáfisis. Además, se menciona que existen a nivel del fémur proximal cinco sistemas de trabéculas que corresponden a las líneas de fuerza mecánicas, siendo estos sistemas los del trocánter mayor, dos principales (uno de tensión y otro de compresión) y dos secundarios (uno de tensión y otro de compresión), Rokwood (1996). Otra descripción menciona que son dos los sistemas trabeculares, uno principal compuesto por dos haces que se expanden sobre el cuello y la cabeza, y otro accesorio con dos haces hacia el trocánter mayor (Kapandji, 1988). Del complejo principal, el primer haz se origina en la cortical externa de la diáfisis y se termina en la parte inferior de la cortical cefálica (Haz arciforme de Gallois y Bosquette) y el segundo haz se expande desde la cortical interna de la diáfisis femoral y la cortical inferior del cuello femoral, y luego se dirige verticalmente hacia la parte superior de la cortical cefálica (haz cefálico o abanico de sustentación) (Kapandji). El complejo o sistema accesorio está formado por dos haces trabeculares que se expanden hacia el trocánter mayor, uno a partir de la cortical interna de la diáfisis (haz trocantéreo) y otro formado por trabéculas verticales paralelas a la cortical externa del trocánter mayor (Kapandji). A causa de la intersección de estos sistemas trabeculares, se originan tres puntos clave en el fémur proximal. El primer punto se encuentra en la meseta trocantérea, donde convergen los haces arciforme y trocantéreo, que al cruzarse forman una clave de arco más densa que desciende de la cortical superior del cuello. El pilar interno es menos sólido y se debilita a medida que aumenta la edad (Kapandji). El segundo punto importante corresponde al centro de rotación de la cadera. Se encuentra en la región medial de la unión entre el cuello y la cabeza. Su sistema trabecular es ojival, formado por la convergencia del haz arciforme y del abanico de sustentación. En la intersección de éstos dos haces, una zona más densa forma

\footnotetext{
Especialista en Ortopedia, Profesor (Invitado) de Anatomía para Posgrado en Medicina. Departamento de Anatomía, Facultad de Medicina, Universidad Nacional Autónoma de México, UNAM. Ciudad de México, México.

*** Especialista en Ortopedia. Profesor Titular del Curso de Ortopedia. American British Cowdray Medical Center, I.A.P. Ciudad de México, México. Profesor de Anatomía para Posgrado en Medicina. Facultad de Medicina, Universidad Nacional Autónoma de México, UNAM. Ciudad de México, México.
} 
el núcleo de la cabeza. Este sistema se apoya en una zona extremadamente sólida, la cortical inferior del cuello femoral que forma el espolón o espina cervical inferior de Merkel, también denominado de Adams o simplemente Calcar (Kapandji; Bartonícek, 2002). Se suele observar en una sección transversal a nivel del trocánter menor y en una sección sagital del fémur proximal como un engrosamiento del hueso intramedular que se extiende desde la parte posterior del cuello hacia la zona posteromedial de la región intertrocantérea, terminando en la cortical posteromedial de la diáfisis femoral proximal (Pablo \& Domínguez, 2002). El calcar tiene una cresta trabecular protruyente y endóstica, que se extiende desde la cortical posteromedial en el cuello femoral hasta la parte distal del trocánter menor y separa a la cavidad femoral del hueso esponjoso dentro del trocánter mayor (Adam et al., 2001). El calcar femoral es un área importante para apoyar y contener los vástagos femorales de los implantes ortopédicos en la cirugía de reconstrucción articular. El tercer punto se encuentra entre el sistema ojival de la meseta trocantérea y el sistema de sustentación cervicocefálico, que es menos resistente, llamado zona de Ward o trígono de Ward. Es un sitio anatómico importante porque es donde se originan las fracturas cervicotrocantéreas en avanzada edad (Kapandji) (Fig. 1).
Biomecánica trabecular. Al aparecer la osteoporosis aunada al aumento de la edad, se manifiesta por una arquitectura trabecular adelgazada con pérdida de su conectividad, una pared cortical con adelgazamiento local, y una porosidad cortical aumentada (Bell et al., 1999a, 1999b; Crabtree et al., 2001; Seeman, 2002; Riggs et al., 2004; Mayhew et al., 2005; Van Rietbergen et al., 2003). Los cambios estructurales trabeculares, al ser progresivos, se han intentado clasificar para correlacionarlos con el riesgo de fracturas o variantes anatómicas (Singh et al., 1970; Dorr et al., 1993). Estos cambios en la arquitectura trabecular y en el hueso cortical, principalmente en el cuello femoral, aumentan el riesgo de fracturas (Bell et al., 1999a, 1999b; Crabtree et al.; Seeman; Riggs et al.; Mayhew et al.). En un estudio de las cadenas trabeculares óseas del fémur proximal en huesos normales y osteoporóticos, se menciona que en éste último, aunque se adapta a las cargas ante la ausencia de trabéculas, se mantiene el aumento del riesgo de fracturas. En el hueso normal las cargas se distribuyen de una manera uniforme en comparación con el hueso osteoporótico y al disminuir la carga para ambos huesos, las magnitudes de las fuerzas se mantienen similares pero con una distribución mucho más amplia en el hueso osteoporótico. Esto disminuye el umbral de tolerancia de carga del hueso normal, fracturas trabeculares a nivel cer-

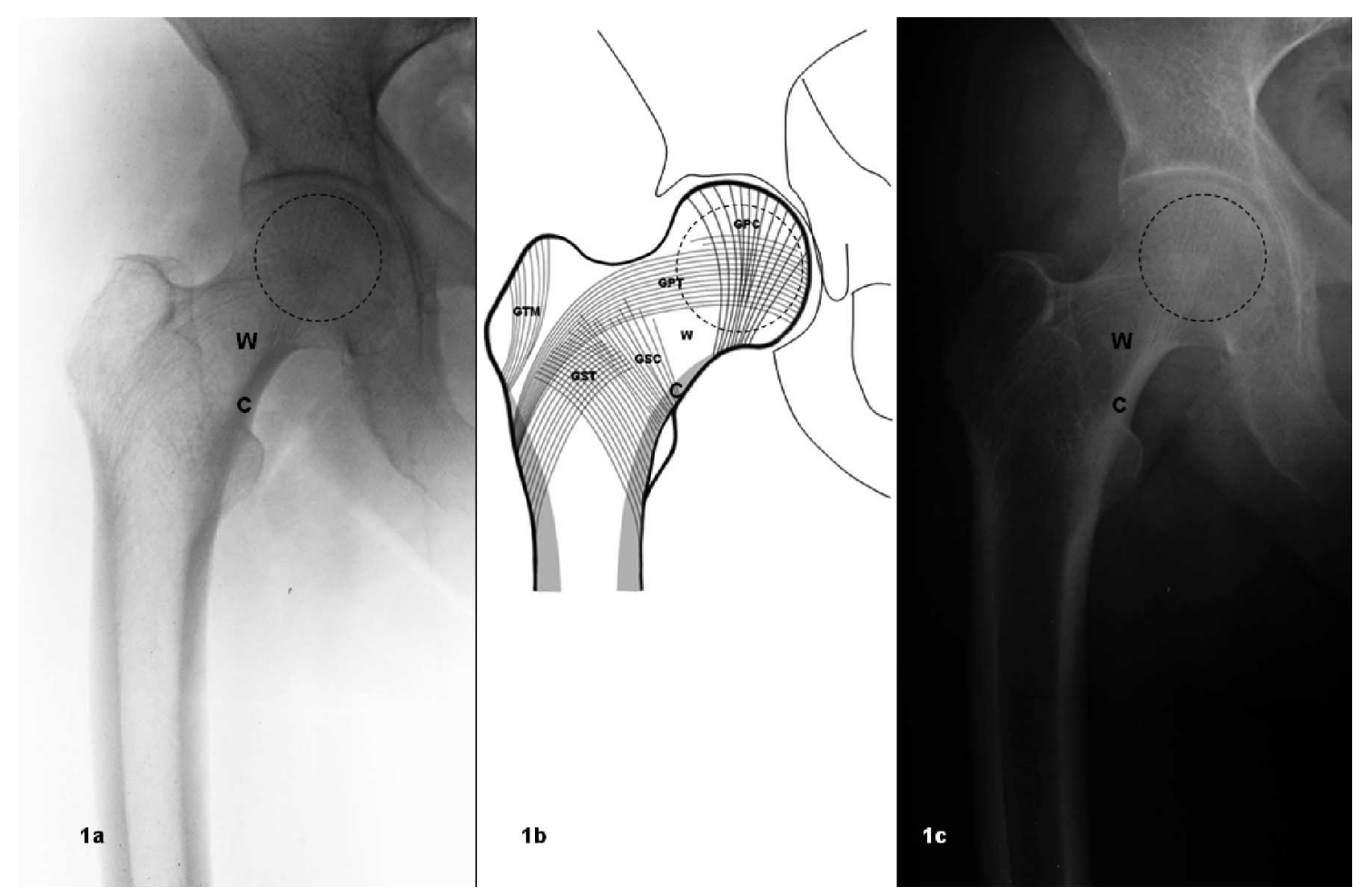

Fig. 1. Radiografía AP de la cadera derecha de un sujeto de 30 años, Recuadro 1a: Radiografía en positivo. Recuadro 1b: Representación esquemática de los grupos trabeculares de tensión y compresión. GTM (grupo del trocánter mayor), GPT (grupo principal de tensión), GPC (grupo principal de compresión), GST (grupo secundario de tensión) y GSC (grupo secundario de compresión). Recuadro 1c: Radiografía en negativo. W = Trígono de Ward. C Calcar. (------) Circunferencia limitando el núcleo de la cabeza femoral. 
vical y sin cambios aparentes en el hueso esponjoso de la cabeza femoral (Van Rietbergen et al.).

Características del hueso subcondral de la cabeza Femoral. La placa de hueso subcondral es una capa de hueso denso que se encuentra por debajo del cartílago articular de las articulaciones sinoviales. Forma la principal estructura de soporte para el cartílago y transmite las cargas desde el cartílago hacia el hueso esponjoso subyacente. La importancia de la interfase entre el cartílago articular y el hueso ha sido reconocida, especialmente con enfoque en la transferencia del estrés desde la flexible matriz del cartílago, hacia el hueso considerablemente más rígido. Se cree que la capa de cartílago calcificado actúa como un intermediario en este proceso (Li \& Aspden, 1997a). En un estudio de las propiedades mecánicas y materiales de la placa de hueso subcondral se mostró que, aunque el grosor de esta capa aumenta en las cabezas con osteoartritis, su rigidez, la densidad del hueso y la masa de fracción de mineral están todas reducidas. En contraste, la placa de hueso subcondral de los pacientes con osteoporosis se encontró más delgada y menos rígida que en los pacientes normales, pero con una composición y densidad muy similares (Li \& Aspden, 1997b). Estos resultados condujeron a la realización de otro estudio acerca de la apariencia microscópica de la placa de hueso subcondral en cabezas femorales. Aquí se encontró que las muestras de pacientes normales y osteoporóticos se presentan con una capa de hueso subcondral recubierta por una capa de cartílago calcificado. En las cabezas femorales provenientes de pacientes con osteoporosis se observó una superficie de fractura más uniforme y un relativo adelgazamiento de las capas, había zonas ocasionales de formación ósea microtrabecular entre las trabéculas del hueso esponjoso subyacente (ausente en los otros grupos) y un mayor número de osteoclastos reabsortivos. La capa de cartílago calcificado estaba casi ausente y la placa ósea estaba aparentemente adelgazada. Con esto se observó que la apariencia de la placa subcondral en el hueso osteoartrítico era muy diferente con respecto al hueso normal y osteoporótico, sugiriendo un fuerte indicativo de una actividad celular anormal (Li et al., 1999).

\section{Características del hueso esponjoso de la cabeza femoral.}

En cuanto al hueso esponjoso de la cabeza femoral, éste se encuentra organizado macroscópicamente por el haz arciforme de Gallois y Bosquette y el haz cefálico o abanico de sustentación, con una zona de intersección donde se forma el punto del núcleo cefálico, como antes se mencionó. Gracias a las nuevas técnicas de reconstrucción tridimensional se pueden observar los detalles microestructurales de los distintos sitios anatómicos y compararlos con los métodos tradicionales histomorfométricos. En una publicación de Hildebrand et al. (1999) se presentaron los detalles microestructurales del hueso esponjoso de distintos sitios anatómicos. Se encontró que la cabeza femoral tiene las mayores fracciones de volúmenes óseos, con hallazgo del grosor de las trabéculas por medición directa de $194 \pm 33 \mu \mathrm{m}$ y la separación trabecular entre $0,45 \mu \mathrm{m}$ a

\section{Lumbar Spine}

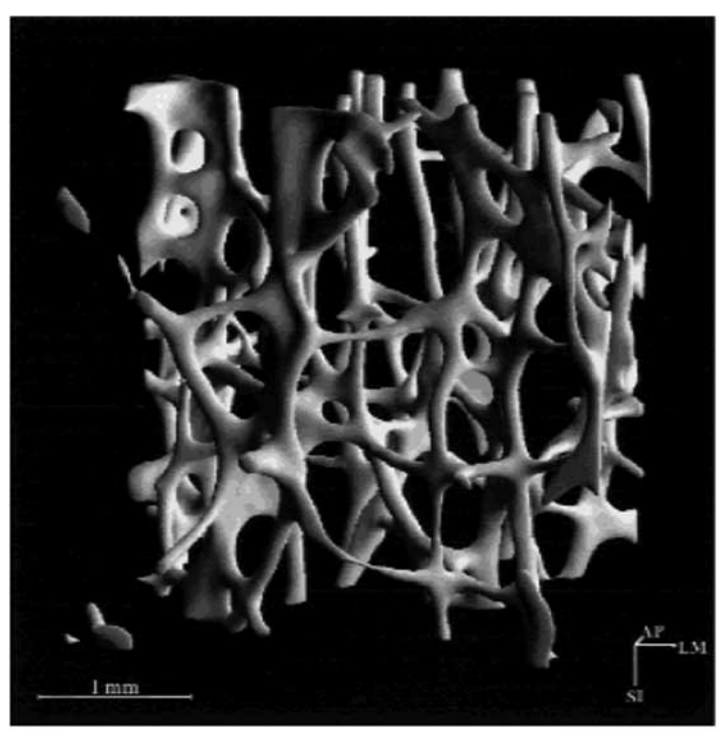

\section{Femoral Head}

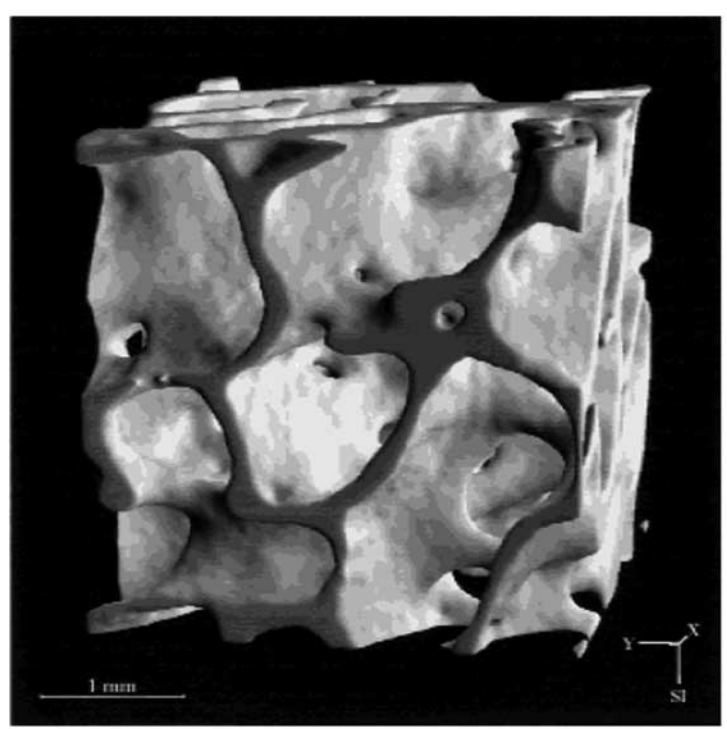

Fig. 2. Estructuras óseas típicas del hueso esponjoso de la columna lumbar y la cabeza femoral. En la columna lumbar dominan las trabéculas parecidas a bastones (Izquierda SMI $=2.5$ ). La estructura trabecular encontrada en la cabeza femoral, sin embargo, es en general más parecida a platos (Derecha, SMI = 0.16). Reproducido de J Bone Miner Res 1999; 14; 1167-1174 (Reproducido con autorización de J. Bone Miner. Res., 14:1167-74, 1999). 
$1,31 \mu \mathrm{m}$. El índice de modelo estructural de la cabeza femoral está caracterizado por placas o platos en lugar de bastones (como en la columna lumbar). En las zonas más densas se encuentran estructuras en forma de plato cóncavo, a veces también referidas como vacíos esféricos (Fig. 2).

Un estudio acerca de los papeles de la microarquitectura y los tipos trabeculares en el módulo de elasticidad del hueso trabecular estudiado por imágenes de $\mu \mathrm{CT}$, ha mostrado que la microarquitectura por si sola afecta el módulo de elasticidad del hueso trabecular y que los platos trabeculares contribuyen mucho más que los bastones al comportamiento elástico óseo (Liu et al., 2006). En otro trapajo publicado se menciona que la distribución de los platos trabeculares y la orientación de los bastones proveen evidencia cuantitativa para la mayoría de los platos trabeculares, estando orientados a lo largo de la dirección principal de carga, mientras que la mayoría de los conos sirven como conexiones transversas entre los platos longitudinales. Sus resultados sugieren que los platos trabeculares dominan globalmente sobre las propiedades elásticas del hueso trabecular (Liu et al., 2008). Es necesario contar con más estudios acerca de estos hallazgos estructurales y biomecánicos para poder explicar cuál de ellos influye en la estabilidad mecánica del hueso. Es importante considerar otras características in vivo tales como la acumulación de daños (microrrompimientos, microfracturas), los enlaces cruzados de colágena y los procesos de mineralización que son difíciles de medir y que pueden también influir sobre la arquitectura ósea (Stauber et al., 2006). En otro estudio de Li \& Aspden (1997a) acerca de las propiedades mecánicas del hueso esponjoso de cabezas femorales de sujetos normales, con osteoartritis y con osteoporosis, se encontró que la densidad aparente y la rigidez mecánica del hueso esponjoso de la cabeza femoral, en comparación con el grupo normal, se encuentran aumentadas en la osteoartritis y disminuidas en la osteoporosis. Sin embargo se mostró también que mientras el hueso con osteoporosis tiene una densidad de material y composición muy similares a las de hueso normal, el de la osteoartritis es menos denso y tiene un contenido de mineral reducido (Li \& Aspden, 1997a).
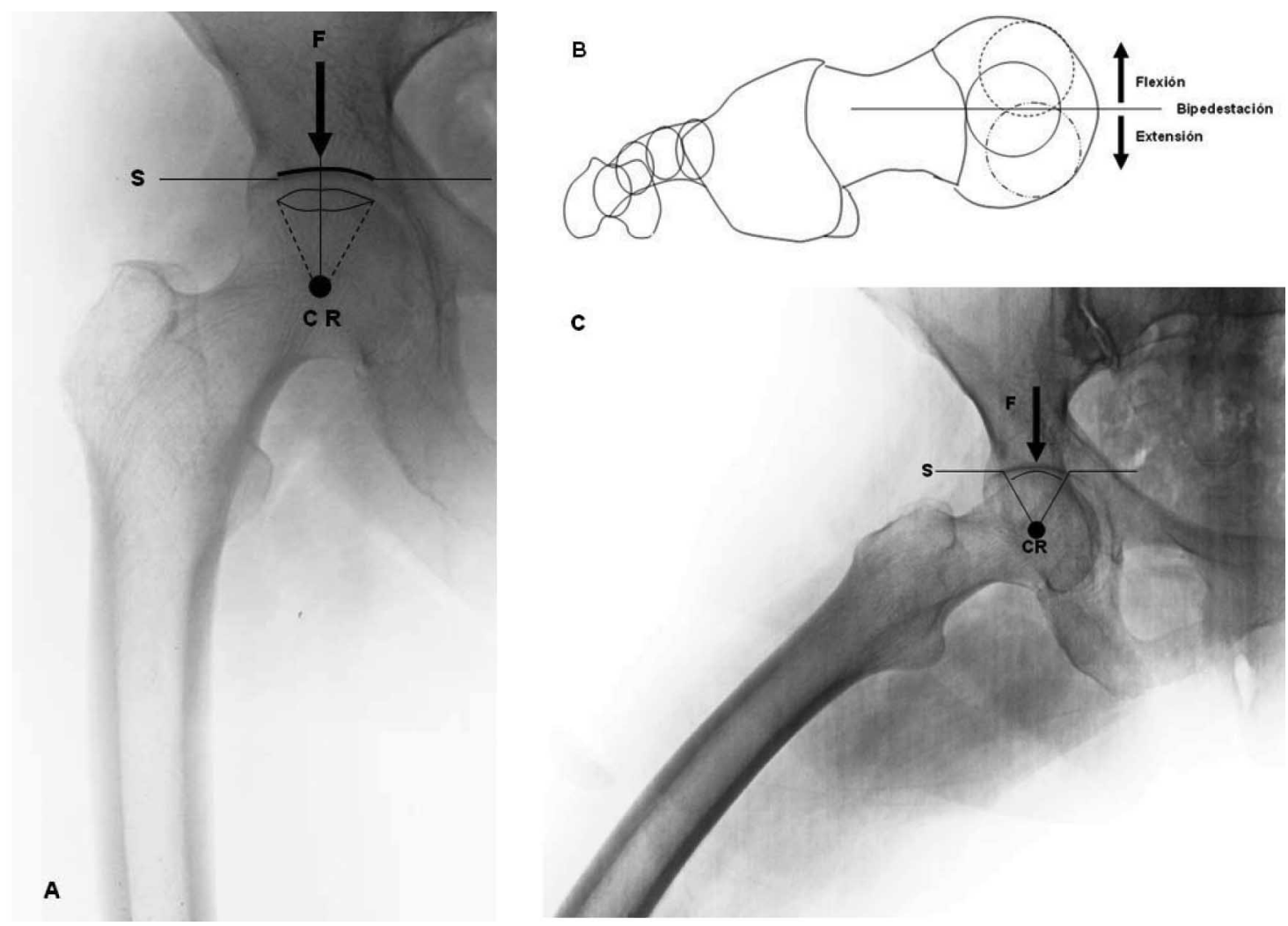

Fig. 3. Zona acetabular y sector esférico femoral. (Recuadro A) Radiografía AP y (Recuadro C) en vista lateral que muestra (S) la zona de carga acetabular o sourcil, (cono) el sector esférico de carga de la cabeza femoral y (CR) el centro de rotación de la cabeza femoral. (F) Vector de fuerza sobre la cadera que representa el peso del cuerpo. (Recuadro B) Representación del fémur proximal en vista superior que muestra al área del sector esférico (superficie de carga) que entra en contacto con la zona de carga acetabular en las diferentes fases de la marcha (Flexión (cadera en rotación lateral con carga supero-anterior y medial), Bipedestación (cadera con rotación neutral y carga superior) y Extensión (cadera con rotación medial con carga supero-posterior y medial]). 
El sector esférico de la cabeza femoral. En la radiografía AP de cadera se puede observar una imagen radiodensa, curva, en la superficie de carga acetabular (sourcil o ceja en francés), descrita por Pauwels en 1963. Representa el hueso subcondral que soporta las cargas compresivas de manera continua y con dirección constante. El área femoral que entra en contacto con el área de carga acetabular, es denominada sector esférico de carga de la cabeza femoral. Este sector se encuentra bajo esfuerzo constante y puede ser proyectado en una radiografía AP de cadera, uniendo los extremos de la ceja acetabular hacia el centro de rotación de la cabeza femoral, siendo éste último su ápex. Se han identificado variantes anatómicas en ésta zona femoral que van desde los $56^{\circ}$ hasta $\operatorname{los} 90^{\circ}$ y pueden dictar el comportamiento de soporte de cargas. Mientras la superficie de área de sector esférico aumenta, la unidad de carga disminuye y viceversa (Bombelli, 1983) (Figs. 3 A y C).

Durante las fases de la marcha existen diferentes secciones de la cabeza femoral afectadas por diversos sectores esféricos. Cada sector tiene su propio centro de estrés pero todos comparten el mismo centro de rotación. Esto a su vez permite establecer dentro de la cabeza femoral una zona afectada por los centros de estrés durante la marcha. Esta área de estrés también se ve afectada por las variantes de superficie del área de carga acetabular y el sector esférico de la cabeza femoral. Entre más ancha sea el área de carga del sector, más cerca se encuentra el centro de estrés al centro de rotación de la cabeza femoral; al contrario, mientras más angosta el área de carga del sector, más cercano está el centro de estrés a la superficie de carga de la cabeza femoral. Cambios en todo lo anterior pueden condicionar el desgaste de las superficies articulares y la osteoartrosis (Bombelli) (Fig. 3 B).

En conclusión, el conocimiento de la microestructura y biomecánica de la cabeza femoral, obliga a que los estudios y observaciones a futuro estén enfocados en determinar mejor los implantes ortopédicos y las técnicas de reconstrucción ósea, con el fin de de detectar tempranamente los datos que desencadenan la enfermedad degenerativa articular o bien mejorar y prolongar la funcionalidad de los implantes.

ÁLVAREZ, S. M. R. \& VELUTINI, K. J. A. Anatomy of the human femoral head: orthopedic issues, part II. Biomechanics and microscopic morphology. Int. J. Morphol., 29(2):371-376, 2011.

SUMMARY: The microscopic structure and biomechanical interactions and characteristics of the femoral head are issues that have been used as scientific support for the understanding of the pathology that affects the hip joint and also for the development of joint replacement implants and bone reconstruction techniques. The aim of this review is to expose the morphological microscopic and biomechanical characteristics of the femoral head in relation to the proximal femur, in order to describe the functional and anatomical aspects, which have made of this structure one of the most important anatomical areas in orthopedic surgery.

KEY WORDS: Femoral head; Proximal femur; Microstructure; Biomechanics; Orthopedics.

\section{REFERENCIAS BIBLIOGRÁFICAS}

Adam, F.; Hammer, D. S.; Pape, D. \& Kohn, D. The internal calcar septum (femoral thigh spur) in computed tomography and conventional radiography. Skeletal Radiol., 30(2):77-83, 2001.

Bartonícek, J. Internal architecture of the proximal femurAdam's or Adams'arch? Historical mystery. Arch. Orthop. Trauma Surg., 122(9-10):551-3, 2002.

Bell, K. L.; Loveridge, N.; Power, J.; Garrahan, N.; Meggit, B. F. \& Reeve, J. Regional differences in cortical porosity in the fractured femoral neck. Bone, 24(1):57-64, 1999a.

Bell, K. L.; Loveridge, N.; Power, J.; Garrahan, N.; Stanton, M.; Lunt, M.; Meggit, B. F. \& Reeve, J. Structure of the femoral head in hip fracture: cortical bone loss in the inferoanterior to superoposterior axis. J. Bone Miner. Res., 14(1):111-9, 1999 b.

Bombelli, R. Biomecánica de la cadera normal, estática y dinámica. Osteoartrosis de la cadera, clasificación y patogénesis. Heildeberg, Berlin, New York, SpringerVerlag, 1983.

Crabtree, N.; Loveridge, N.; Parker, M.; Rushton, N.; Power, J.; Bell, K. L.; Beckt, T. J. \& Reeve, J. Intracapsular hip fracture and the region-specific loss of cortical bone: analysis by peripheral quantitative computed tomography. J. Bone Miner. Res., 16(7):1318-28, 2001.

Dorr, L. D.; Faugere, M. C.; Mackel, A. M.; Gruen, T. A.; Bognar, B. \& Malluche, H. H. Structural and cellular 
assessment of bone quality of proximal femur. Bone, 14:231-242, 1993.

Hildebrand, T.; Laib, A.; Müller, R.; Dequeker, J. \& Rüegsegger, P. Direct three-dimensional morphometric analysis of human cancellous bone: microstructural data from spine, femur, iliac crest and calcaneus. J. Bone Min. Res., 14(7):1167-74, 1999.

Kapandji, I. A. Cuadernos de fisiología articular. 4ª Ed. Barcelona, Masson, 1988.

Li, B. \& Aspden, R. M. Composition and mechanical properties of cancellous bone from femoral head of patients with osteoporosis or osteoarthritis. J. Bone Miner. Res., 12(4):641-51, 1997a.

Li, B. \& Aspden, R. M. Mechanical and material properties of the subcondral bone plate from the femoral head of patients with osteoarthritis or osteoporosis. Ann. Rheum. Dis., 56(4):247-54, 1997b.

Li, B.; Marshall, D.; Roe, M. \& Aspden, R. M. The electron microscope appearance of the subchondral bone plate in the human femoral head in osteoarthritis and osteoporosis. J. Anat., 195(Pt 1):101-10, 1999.

Liu, X. S.; Sajda, P.; Saha, P. K. ; Wehrli, F. W.; Bevill, G. ; Keaveny, T. M. \& Guo, X. E. Complete volumetric decomposition of individual trabecular plates and rods and its morphological correlations with anisotropic elastic moduli in human trabecular bone. J. Bone Miner. Res., 23(2):223-35, 2008.

Liu X. S.; Sajda, P.; Saha, P. K.; Wehrli, F. W. \& Guo, X. E. Quantification of the roles of trabecular microarchitecture and trabecular type in determining the elastic modulus of human trabecular bone. J. Bone Miner. Res., 21(10):1608-17, 2006.

Mayhew, P. M.; Thomas, C. D.; Clement, J. G.; Loveridge, N.; Beck, T. J.; Bondield, W.; Burgoyne, C. J. \& Reeve, J. Relation between age, femoral neck cortical stability, and hip fracture risk. Lancet, 366(9480):129-35, 2005.

Pablo, de L. C. \& Domínguez, E. Tratamiento de las fracturas de huesos largos. Manual de Osteosíntesis. Madrid, Masson, 2002. pp.88-97.

Riggs, B. L.; Melton, I. L. J. $3^{\text {rd }}$; Robb, R. A.; Camp, J. J.; Atkinson, E. J.; Peterson, J. M.; Rouleau, P. A.; McCollough, C. H.; Bouxsein, M. L. \& Khosla, S. Population-based study of age and sex differences in bone volumetric density, size, geometry, and structure at different skeletal sites. J. Bone Miner. Res., 19(12):1945-54, 2004.

Rokwood, C. A.; Green, D. P.; Bucholz, R. W. \& Heckman, J. D. Fracturas del cuello femoral. Rokwood \& Green's fractures in adults. $4^{\text {a }}$ Ed. Philadelphia, LippincottRaven, 1996. p.1667.

Seeman, E. Pathogenesis of bone fragility in women and men. Lancet, 359(9320):1841-5, 2002.

Singh, M.; Nagrath, A. R. \& Maini, P. S. Changes in trabecular pattern of the upper end of the femur as an index of osteoporosis. J. Bone Joint Surg. Am., 52(3):457-67, 1970.

Stauber, M.; Rapillard, L.; van Lenthe, G. H.; Zysset, P. \& Müller, R. Importance of individual rods and plates in the assessment of bone quality and their contribution to bone stiffness. J. Bone Miner. Res., 21(4):586-95, 2006.

Van Rietbergen, B.; Huiskes, R.; Ekcstein, F. \& Rüegsegger, P. Trabecular bone tissue strains in the healthy and osteoporotic human femur. J. Bone Miner. Res., 18(10):1781-8, 2003.

Dirección para correspondencia:

Raúl Álvarez San Martín

Especialista en Ortopedia

Profesor (Invitado) de Anatomía Posgrado en Medicina

Departamento de Anatomía

Facultad de Medicina

Universidad Nacional Autónoma de México, UNAM.

Calle B. Manzana X. No.19 Col. Educación

CP 04400

México D.F. Del. Coyoacán

Ciudad de México

MÉXICO

Email: alvarez_sanmartin@yahoo.com.mx

Recibido : 16-01-2010

Aceptado: 08-11-2010 